\title{
Die Konstruktion würde+Infinitiv als evidentieller Ausdruck des Deutschen
}

\begin{abstract}
Artykuł otwiera definicja ewidencjalności jako kategorii semantycznej oraz krótki przegląd wyrażeń języka niemieckiego, które mogłyby być uznane za wykładniki ewidencjalności. Następnie w nawiązaniu do pracy SMIRNOVEJ (2006) zaproponowany zostaje opis semantyczny konstrukcji würde+bezokolicznik, uwzględniający komponenty modalne oraz ewidencjalne (zarówno z zakresu ewidencjalności reportatywnej jak i inferencyjnej), w różnych konfiguracjach aktywizowane przez kontekst konkretnego użycia tej konstrukcji.

Der Artikel beginnt mit einer Definition der Evidentialität als semantischer Kategorie und einer kurzen Übersicht sprachlicher Ausdrücke, die als Evidentialitätsmarker des Deutschen angesehen werden können. Anschließend wird - in Anlehnung an SMIRNOVA (2006) - eine Analyse der Konstruktion würde+Infinitiv vorgeschlagen, der zufolge ihre Grundbedeutung modale und evidentielle (sowohl quotative als auch inferentielle) Bestandteile vereint, auch wenn nicht immer alle zugleich durch den Verwendungskontext aktiviert werden.

The article begins with a definition of the evidentiality as a semantic category and a short overwiew of linguistic expressions which can be seen as evidentiality markers in German. Then, with reference to SMIRNOvA (2006), a semantic view on the construction würde+infinitive is proposed, in which its meaning contains both modal and evidential (quotative as well as inferential) components, which, however, are not activated at the same time by the context of its use.
\end{abstract}




\section{Zur Definition der Evidentialität}

In BUSSMANN (2002:206) wird die Evidentialität definiert als eine ,strukturelle Dimension der Grammatik, deren Werte von Konstruktionstypen ausgedrückt werden, die die Quelle der Information kodieren, die ein Sprecher weitergibt“". Traditionellerweise wird ,der Terminus Evidentialitätsmarker nur auf grammatische Elemente angewendet [...], [...] folglich fallen lexikalische Einheiten oder syntaktische Konstruktionen mit vergleichbarer Bedeutung nicht unter diesen Begriff“ (DIEWALD 2004:235; s. auch AIKHENVALD 2006:6; FALLER 2007:224). DENDALE / TASMOWSKI (2001:341) halten die Frage, inwieweit die Evidentialitätsmarker jeweils eine grammatikalisierte morphologische Kategorie darstellen, für eine der wichtigsten in der gegenwärtigen Evidentialitätsforschung. LAZARD (2001:360) unterscheidet zwischen Sprachen, in denen die Evidentialität eine obligatorische Markierung mit einem gebundenen Morphem am Verb darstellt (z.B. Tuyuca), und Sprachen, die ein verbales Paradigma besitzen, mit dessen Hilfe die Evidentialität optional zum Ausdruck gebracht werden kann und folglich in Opposition zur Nicht-Markierung steht. Zur letzten Gruppe gehören die meisten in Südosteuropa und in Westasien gesprochenen Sprachen. Neben ,evidentiality as a single tightly knit and coherent category“ unterscheidet AIKHENVALD (2006:9) evidentielle Erweiterungen nicht-evidentieller Kategorien sowie evidentielle Strategien (,evidential strategies'), d.h. „categories and forms which acquire secondary meanings somehow related with information source [...]. They are distinct from evidentials proper, whose primary - and not infrequently exclusive - meaning is information source" (AIKHENWALD 2006:105, 277). Ferner gibt es eine wachsende Gruppe von Linguisten, die explizit von ,lexical markers of evidentiality“ (WIEMER 2005:107) sprechen (s. DE HAAN 2001:194; HASSLER 2002:143; SMIRNOVA 2006:61) und die Beschränkung der semantischen Domäne auf grammatische Ausdrucksmittel kritisieren:

[...] since we are dealing with notional distinctions which can in principle be expressed by either lexical or grammatical means (or by both at once), I do not understand why research into lexical coding of evidentiality (and its empirical correlation with epistemic modality) should not commence before we will have fully understood which kinds of evidentiality systems exist in the grammars of world's languages. (WIEMER 2005:109)

Dementsprechend beschäftigt sich HASSLER (2002:161) u. a. mit der evidentiellen Bedeutung französischer Modalverben, Wahrnehmungsverben, Kommunikationsverben, Präpositionen, Tempus- und Modusformen und Partikeln, 
während WIEMER (2006:5) ,[p]articles, parentheticals, conjunctions and prepositions as evidentiality markers in contemporary Polish" untersucht. Im Folgenden schließe ich mich dieser Betrachtungsweise an.

In semantischer Hinsicht kann die Evidentialität je nach der Art der ausgedrückten Informationsquelle untergliedert werden.

Evidentiality is traditionally divided in two main categories: direct evidentiality, which shows that the speaker has directly witnessed the action, and indirect evidentiality, which shows that the speaker has no direct evidence for his/her statement, but has other sources for making the statement. Typical direct evidential categories are visual and auditory evidence, stating that the speaker has respectively seen and heard the action. Indirect evidentials can be inferentials, which mean that the speaker has inferred the action from available evidence, and quotatives (also referred to in the literature as reportive or hearsay evidentiality), which states that the speaker knows about the event from being told by another person. (DE HAAN 2005:379)

DIEWALD $(2004,2005)$ postuliert direkte Evidentialität in der Bedeutung der deutschen Verben scheinen und werden (in Verbindung mit einem Infinitiv). Häufiger werden jedoch im Deutschen Bedeutungen aus dem Bereich der indirekten Evidentialität (sowohl der Inferentialität als auch der Quotativität) beschrieben (vgl. BUSSMANN 2002:206), auf die in Abschnitt 2 näher eingegangen wird. Die Evidentialität muss von der epistemischen Modalität unterschieden werden:

Evidential meanings [...] specify the source of knowledge by virtue of which the speaker feels entitled to make a statement. Epistemic modality is different, because it refers to the actual speaker's subjective assessment of the veracity of his assertion(s), i.e. to the speakers evaluation of the uttered proposition(s) as being true, likely or not true. (WIEMER 2006:7)

Eine saubere begriffliche Trennung der beiden semantischen Bereiche, die auch von DE HAAN (1999:84,98), NUYTS (2001:27) und AIKHENVALD (2006:7) postuliert wird, ist umso wichtiger, als evidentielle Bedeutungen in den meisten indoeuropäischen Sprachen (auch im Deutschen) typischerweise durch sprachliche Mittel ausgedrückt werden, die auch epistemische Modalität markieren können. ${ }^{1}$ Dies trifft auch auf die meisten deutschen Ausdrücke zu, für die man eine evidentielle (inferentielle oder quotative) Bedeutung annimmt.

Aus diesem Grund wurde die Evidentialität von vielen - insbesondere älteren Autoren als ein Teilbereich der Modalität angesehen (z. B. WILlET 1988; PALMER 2001), oder man bezog den Terminus ,Evidentialität‘ sowohl auf die Markierung der Quelle einer Information als auch auf die Bewertung ihrer Faktizität (vgl. CHAFE 1986:262). 


\section{Evidentialität im Deutschen}

\subsection{Inferentielle Evidentialität}

\subsubsection{Modalwörter}

Die deutschen Modalwörter anscheinend, offenbar und offensichtlich werden in Helbig / Helbig (1990) als ,Hypotheseindikatoren“ bezeichnet. Ihre Bedeutungsangaben enthalten Phrasen wie ,,auf Grund von äußeren Wahrnehmungen oder erkenntnismäßig zu erschließenden Bedingungen“ (HELBIG / HELBIG 1990:176, 179). Auch die Bedeutungsexplikationen von sogenannten Gewissheitsindikatoren - offenkundig, evidentermaßen, augenscheinlich und sichtlich - suggerieren inferentielle Bestandteile, die jedoch nicht explizit als solche behandelt werden.

(1) Die Kaufhalle hat anscheinend gerade erst geöffnet. (HELBIG / HELBIG 1990:80)

(2) Die Ausgrabungen stammen (ganz) offenkundig aus der Bronzezeit. (HELBIG / HELBIG 1990:179)

Es gibt bislang auch keine Untersuchung zu lexikalischen Evidentialitätsmarkern im Deutschen. Für das polnische Modalwort widocznie nimmt WIEMER (2006:53) an, dass seine inferentielle Bedeutungskomponente eine syllogistische Struktur hat, die sich wie folgt paraphrasieren lässt: ,,[...] I have perceived (or: I am perceiving) something that lets me know that Q. [...] Since I know Q, $\mathrm{P}$ must be true. [...].“ Ferner kann scheinbar, dessen Bedeutung eine inferentielle Komponente und zugleich die Information enthält, dass die betreffende Schlussfolgerung falsch ist, zu den Markern der Inferenzialität gerechnet werden (WIEMER 2006:61). ${ }^{2}$

(3) Sie trat bei der Verteidigung scheinbar ruhig und sicher auf. (HELBIG / HELBIG 1990:189)

\subsection{2 müssen, dürfte}

Die Modalverben müssen und dürfte ${ }^{3}$ in Sätzen wie Hierbei muss/dürfte es sich um eine Verwechslung handeln werden in BUSSMANN (2002:206) entsprechend ,als starke vs. schwache Inferential-Kennzeichnung“ bezeichnet.

$2 \quad \mathrm{Zu}$ beachten ist allerdings, dass scheinbar polysem ist. In seiner zweiten Bedeutung ist das Modalwort synonym mit anscheinend (HeLBIG / HeLBIG 1990:189).

3 Das Verb dürfen kann diese Bedeutung nur im Konjunktiv II haben. 
Für DIEWALD (2004) haben diese Verben dagegen eine rein epistemisch-modale Bedeutung. Dürfte verbinde die unsichere Faktizitätsbewertung mit einem anaphorischen Verweis auf ein Faktizitätsurteil. Die Bedeutung von dürfte wird also zwar als ,ich folgere daraus, dass es wahrscheinlich ist, dass“" (DIEWALD 2004:242f.) paraphrasiert, doch überraschenderweise gar nicht als inferentiell betrachtet. Bei müssen handele es sich um eine ,rein deiktische Faktizitätsbewertung [...], wobei [...] müssen eine Tendenz zum Wert [+faktisch] signalisiert“ (DIEWALD 2004:240). Nach VAN DER AUWERA / PLUNGIAN (1998:85f.) fällt - in Sprachen mit grammatischen inferentiellen Ausdrücken - inferentielle Evidentialität mit der epistemischen Notwendigkeit bedeutungsmäßig zusammen (bei beiden handelt es sich um die Sicherheit eines Urteils aufgrund von anderen Urteilen), weswegen solche Ausdrücke oft mit dem epistemischen must ins Englische übersetzt werden. WIEMER (2006:19) vermutet, dass auch dem polnischen Verb musieć eine evidentielle Bedeutung zugeschrieben werden muss. DE HAAN (1999:87f.) sieht das englische must in Sätzen wie You must have been home last night als epistemisch, weil es nur aufgrund der epistemischen Bedeutungskomponente ,Sicherheitsgrad eines Urteils' in Opposition zu z. B. may tritt. Weil aber für die Einschätzung dieses Grades die vorhandene Evidenz eine wesentliche Rolle spielt, kann sich die evidentielle Bedeutung aus der epistemischen aufgrund einer konversationellen Implikatur entwickeln. Genau dies geschehe beim niederländischen Verb moeten, das in geeigneten Kontexten ausschließlich inferentiell verstanden werden kann: ,When the hearer hears a sentence like [He moet een goede film zijn] he or she can decide that the presence of evidence for the speaker's statement is more important than the evaluation." (DE HAAN 1999:89)

Für das deutsche müssen zeichnet SMIRNOVA (2006:96f.) einen vermutlichen Entwicklungsweg der evidentiellen Bedeutungskomponente aus einer ursprünglichen konversationellen Implikatur nach:

Aus der relativ hohen Sicherheit des Sprechers gegenüber dem Ausgesagten [...], die z.B. das deiktisch gebrauchte deutsche Modalverb müssen zum Ausdruck bringt, ergeben sich hörerspezifische Implikaturen [...], dass der Sprecher für die Behauptung, für die Zuweisung eines hohen Faktizitätsgrades ,Gründe' (=Evidenzen) hat, und ferner, dass der Sprecher die Proposition aus diesen ,Gründen ‘ schlussfolgert. Diese im evidentiellen Sinne gedeuteten Implikaturen [...] können allmählich von den Sprechern als inhärente Bedeutungskomponente des Zeichens empfunden werden. Diese Phase, in der evidentielle und modale Bedeutungen soweit miteinander verschmolzen sind, dass sie untrennbar voneinander empfunden werden, ist im Deutschen noch nicht erreicht, zumeist unterliegt evidentielle Interpretation z. B. des Verbs müssen bestimmten Restriktionen [...]. 


\subsection{3 drohen, versprechen, scheinen}

Den Modalitätsverben scheinen, drohen und versprechen (vgl. 4-6) schreibt DIEWALD (2004:238-250) eine ,zusätzliche evidentielle Komponente“ zu. Dabei verbinde scheinen eine unsichere Faktizitätsbewertung mit dem Verweis auf direkte visuelle Evidenzen als deren Quelle. Seine Bedeutung lasse sich folglich mit ,aufgrund visueller Information vermute ich, dass $p^{\text {“ paraphrasie- }}$ ren. Gegenwärtig sei das Verb scheinen jedoch dabei, ,sich zu einem unspezifischen Evidentialitätsmarker zu entwickeln“ mit der Bedeutung ,aufgrund von nicht weiter spezifizierten Evidenzen vermute ich, dass $p$ “. Dieselbe Paraphrase treffe auch auf drohen zu, dessen Bedeutung allerdings zusätzlich eine „ingressive und negativ wertende Komponente“ enthalte: Die Konstruktion könne nur auf erst in der Zukunft zu erwartende und zugleich unerwünschte Sachverhalte angewendet werden. Versprechen verweise ebenfalls auf eine unspezifische Informationsquelle und auf noch ausstehende Sachverhalte, die allerdings positiv gewertet werden.

(4) Das scheint man in der Landesgeschichte aufgespürt zu haben. (DIEWALD 2004:246)

(5) In Ungarn drohen die Dämme zu brechen. (DIEWALD 2004:248)

(6) Ausgerechnet als mein Traum versprach, pikant zu werden, erwachte ich. (DIEWALD 2004:250)

\subsection{4 werden, würde}

In DiEwALD (2005:30f.) wird auch der Konstruktion werden+Infinitiv eine evidentielle Bedeutung zugeschrieben, die allerdings durch die lexikalische Bedeutung von werden um eine dynamische Komponente angereichert sei:

Der Sprecher verweist auf Indizien für eine Entwicklung, deren Ergebnis der im Satz dargestellte Sachverhalt ist. Dieser Endzustand ist noch nicht erreicht, die Indizien gelten dem Beginn der Veränderung. Eine tentative Paraphrase für diese evidentielle Grundbedeutung von werden \& Infinitiv ist: [...] Der Sprecher hat direkte Anzeichen dafür, dass eine Veränderung vor sich geht, die zu p führt.

Von dieser Basisbedeutung und den kontextuellen sowie situativen Faktoren lassen sich nach DIEWALD (2005:31) alle Lesarten der Konstruktion ableiten. SMIRNOVA (2006:103-105,109-131) übernimmt diese Sichtweise, modifiziert sie aber in einem wichtigen Punkt: Die Konstruktion werden+Infinitiv drücke keine direkte, sondern eine indirekte, genauer: inferentielle Evidentialität aus.

M.E. ist es in erster Linie die Schlussfolgerung des Sprechers, die mit der Konstruktion werden+Infinitiv kodiert wird. [...] Werden kodiert aufgrund seiner ur- 
sprünglichen aktionalen Semantik eine Ausgangspunkt-Ziel-Relation, die im Laufe der Grammatikalisierung als eine Grund-Folge-Relation gedeutet wird. Die Informationsquelle, die Evidenzen, die dem Sprecher vorliegen, bilden den Ausgangspunkt oder den Grund für eine logische Operation des Schließens, die den Sprecher letztendlich zu einer Folgerung führt, die mit werden-Konstruktionen ausgedrückt wird. Die Evidenzen werden nicht weiter spezifiziert und nicht explizit genannt. (SMIRNOVA 2006:104)

Der Satz Er wird jetzt im Büro sitzen lasse sich also paraphrasieren als „Ausgehend von nicht weiter spezifizierten Informationen (Evidenzen) schließt (folgert) der Sprecher, dass p [er sitzt im Büro]“" (SMIRNovA 2006:128). Beide Ansätze fügen sich in die Beschreibungstradition, in der der werden-Periphrase eine Basisbedeutung zugeschrieben wird, die weder rein temporal noch rein modal ist, sondern die Subjektivität des Sprechers betont. ${ }^{4}$ In diesem Sinne sieht SMIRNOVA (2006:105) die inferentielle Evidentialität als eine deiktische Kategorie: werden+Infinitiv „bringt den entfernten, „origoexklusiven“ deiktischen Wert [+INDIREKT] zum Ausdruck mit dem zusätzlichen Verweis auf den aktuellen Sprecher [+SUBJEKTIV]“. Die Bedeutung der werden-Periphrase lasse sich als eine gerichtete Relation darstellen. Ihren Ausgangspunkt bildet eine Informationsquelle, die ,unabhängig vom Sprecher [existiert], [...] aber vom Sprecher als Urheber der Äußerung in den sprachlichen Kontext hereingebracht [wird]“". Ihr Zielpunkt ist die assertierte Proposition, während „[d]er Pfad oder Relator werden den (mentalen) Weg von der sprachlich formulierbaren Informationsquelle (Prämissen) zur sprachlich ausgedrückten Proposition (Folgerung) [kodiert]“ (SMIRNOVA 2006:127). Dieselbe Bedeutungsstruktur werde auch in der Konstruktion würde+Infinitiv realisiert. Sie könne allerdings - abhängig vom Verwendungskontext - entweder ,dominant gesetzt“ oder zugunsten von anderen Bedeutungskomponenten „eliminiert“ werden, wodurch verschiedene Lesarten dieser Konstruktion entstehen. ${ }^{5}$

\subsection{Quotative Evidentialität}

\subsubsection{Modalwörter und Präpositionen}

Von quotativer Bedeutung lässt sich bei den deutschen Präpositionalphrasen vom Typ laut $x, x$ zufolge sowie den Modalwörtern angeblich und (dem kaum gebräuchlichen) vorgeblich reden. Im Vergleich zum Englischen (mit appa-

4 Ihre Auffassung sieht SMIRNOva (2006:104f.) durch die Ansichten von MARSCHALl (1987) und MoRTELMANS (2004) bestätigt, während DiEWALD (2004:30) ihren Ansatz als eine Entwicklung desjenigen von FRITZ (2000) versteht.

5 Vgl. SMiRnOva (2006:131f., 240) sowie auch den Abschnitt 3 unten. 
rently, supposedly, allegedly, reportedly, vgl. WIERZBICKA 2006:278-284) und zum Polnischen (das über podobno, niby, jakoby und rzekomo verfügt, vgl. WIEMER 2006), erscheint das Deutsche als eine an quotativen Modalwörtern ausgesprochen arme Sprache.

\subsection{2 sollen und wollen}

Die Verben wollen und sollen in Sätzen wie Philipp will / soll ein direkter Nachfahre Karls des Großen sein werden u. a. in BUSSMANN (2006:206) ,als Subjekt- und 3. Person-Quotativ-Markierung“ bezeichnet (s. auch DE HAAN 1999:93; PALMER 2001:9). Nach AIKHENVALD (2006:150) handelt es sich beim quotativen Gebrauch von sollen um einen Grenzfall zwischen lexikalischer Evidentialitätsmarkierung und einer evidentiellen Strategie. DIEWALD (2004:41) bezeichnet die beiden Verben zwar als Quotative, rechnet sie aber nicht zu den evidentiellen Ausdrücken. Die letzteren (hearsay evidentials bzw. reportives) liegen nach ihrer Auffassung nur dann vor, wenn

[...] der Schwerpunkt eher auf dem Inhalt des Mitgeteilten und der Tatsache [liegt], dass der Sprecher diese Information durch andere Sprecher in sprachlicher Form erfahren hat [...]. Unter Umständen wird in diesem Fall die Information, dass es sich um ein Zitat handelt, das gehört wurde, irrelevant; fokussiert wird stattdessen die Tatsache, dass das Wissen des Sprechers indirekt durch kommunikative Akte erworben wurde und deshalb möglicherweise einen unsicheren Faktizitätsgrad hat.

Im Gegensatz dazu besteht nach DIEWALD (2004:41) die definitorische Eigenschaft von Quotativen darin, dass sie ,den Zitatcharakter der mitgeteilten Information [betonen]“, wobei auch die eventuell ausgedrückte Faktizitätsbewertung immer die des originalen Sprechers ist, während sich der zitierende Sprecher seiner eigenen enthält. Mit Bezug auf sollen oder wollen ist eine solche Abgrenzung jedoch insoweit problematisch, als mit ihrer Hilfe keinesfalls immer genaue Zitate formuliert werden. Nach ZIFONUN (1997:1896) können vielmehr einem Satz wie (7a) auch Äußerungen wie (7b) oder (7c) zugrunde liegen. Ähnlich müsse die Person, auf die sich das Subjekt des Satzes (8) bezieht, nicht geäußert haben: Ich habe nichts davon gewusst. Es genüge, wenn sie auf eine beliebige Weise versucht, den Sprecher von (8) dies glauben zu lassen.

(7) (a) Dem Vernehmen nach sollen sich die Geiseln noch in Teheran befinden.

(b) Die Geiseln müssen sich noch in Teheran befinden. 
(c) Es gibt keine Informationen über eine Veränderung der Lage in der Geiselaffäre. (ZIFONUN 1997:1893)

$$
\text { Er will nichts davon gewusst haben. }
$$

Auch in der generellen Verbindung von reportiver Evidentialität mit einer obligatorischen epistemisch-modalen Einstellung stimmen die meisten Forscher mit Diewald nicht überein. Als evidentiell werden auch Ausdrücke angesehen, bei denen die epistemische Einstellung fehlt bzw. nur in einer Distanzhaltung (der Sprecher übernimmt keine Verantwortung für die Wahrhaftigkeit der Aussage) besteht (AIKHENVALD 2006:33, 108; WIEMER 2006:7). AIKHENVALD (2006:25, 50) nimmt zwar auch eine Unterscheidung zwischen ,reported evidentials ' und ,quotatives' vor, rechnet die letzteren jedoch ebenfalls zu den Evidentialitätsmarkern. Der Terminus ,reported evidentials` bezieht sich bei ihr auf die Kennzeichnung von Informationen aus nicht weiter spezifizierten verbalen Quellen, der Terminus ,quotatives‘ auf Informationen, die von einer bestimmten Person stammen. In einigen Indianersprachen Nordamerikas läuft diese Differenzierung auf jeweils unterschiedliche Markierung indirekt verbal erworbener Informationen einerseits und direkter Zitate andererseits (Comanche), oder von einfach Berichtetem und Mythischem hinaus (Tonkawa, vgl. AIKHENWALD 2006:51). Ähnlich differenzierte bereits ANDERSON (1986:289) zwischen , ,...] at least four kinds of reportives: (a) hearsay, (b) general reputation, (c) myth and history (these three being evidentials), and (d) ,quotative " (marginally ,evidential')“. SMIRNOVA (2006:83f.) sieht den Grund für den nur marginal evidentiellen Status der ,quotatives“ darin, dass ,ihre Bedeutung nicht primär im Denotieren einer Informationsquelle zu suchen ist“. Sie selbst lässt die Frage der Abgrenzung zwischen ,reportives“ und ,den sog. ,Quotativen ", ferner ihre[r] Einordnung in evidentielle oder modale Systeme“ offen, da sie für ihre Fragestellung irrelevant sei und ,in der heutigen linguistischen Forschung noch diskutiert" werde. Im vorliegenden Aufsatz wird nicht zwischen den beiden Termini unterschieden, vielmehr wird nur einer benutzt: ,Quotativ“ nenne ich diejenigen sprachlichen Mittel, die als Informationsquelle eine verbale Mitteilung durch eine vom aktuellen Sprecher verschiedene Instanz ausweisen.

\subsubsection{Quotativer Konjunktiv}

FABRICIUS-HANSEN (2005:522) nennt das „Referat“ bzw. die ,indirekte Redewiedergabe“ (529f.) als eine der beiden wichtigsten Funktionen des markierten Modus des Deutschen, d.h. des Konjunktivs. ZIFOnUN (1997:1753) folgend, spricht sie vom, Indirektheitskonjunktiv‘. Traditionellerweise zählt 
man ihn neben einem einleitenden Ausdruck des Sagens (wie sagen, erzählen, berichten etc.) und subordinierenden Konjunktionen (dass, ob etc.) zu den sprachlichen Mitteln, die das Vorliegen einer Redewiedergabe im weiteren Sinne signalisieren.

Zur formalen Kennzeichnung der indirekten Rede dienen:

1. der Konjunktiv

2. redeeinleitende Verben

3. die Nebensatzform

Keines dieser Mittel ist obligatorisch, doch ist gewöhnlich zumindest eines vorhanden, um die indirekte Rede als solche zu kennzeichnen. (HELbIG / BusCHA 2001:174)

Die Redewiedergabe einerseits und die Mittel der quotativen Evidentialität andererseits dienen prinzipiell demselben Zweck: Sie bringen zum Ausdruck, dass eine andere Person die Quelle der betreffenden Information ist (vgl. AIKHENVALD 2006:135). Deswegen ist es nicht verwunderlich, dass der deutsche Indirektheitskonjunktiv in Überblicksdarstellungen zur Evidentialität behandelt wird. Insbesondere handelt es sich um seinen Gebrauch in desubordinierten Hauptsätzen, in denen er als einziger Redewiedergabemarker auftritt. Textpassagen, die aus mindestens einem solchen Satz bestehen (vgl. die eingeklammerte Passage in Beispiel 9) nennt man ,berichtete Rede" (FABRICIUSHANSEN 2005:530; THIEROFF 1992), , freie indirekte Rede ' (RONCADOR 1988) bzw. ,de-subordinated free indirect discourse' (AIKHENVALD 2006:134).

(9) Miks bestritt natürlich alles. [Von dem Bock wisse er nichts. Er habe nur Krähen schießen wollen, und das könne unmöglich ein großes Verbrechen sein.] (Zit. in AIKHENVALD 2006:107)

Aikhenvald zählt den Konjunktiv in berichteter Rede zu den evidentiellen Strategien. Den Ausgangspunkt der Grammatikalisierung bildet dabei der $\mathrm{Zu}-$ stand, in dem der Konjunktiv einen obligatorischen Modus in Komplementsätzen von nichtfaktiven Verba dicendi darstellt und dadurch das Vorliegen der indirekten Rede mit signalisiert. ${ }^{6}$ Später kann er nicht nur in Komplementsät-

6 Bis ins Frühneuhochdeutsche bestand keine Opposition zwischen dem Konjunktiv I (Konjunktiv Präsens) und Konjunktiv II (Konjunktiv Präteritum). Vielmehr wurde ihre Distribution durch das Tempus des übergeordneten Verbs bestimmt: „Nach Verben im Präteritum stand der Konjunktiv Präteritum, und nach Verben im Präsens stand der Konjunktiv Präsens“" (WelKe 2005:463). Nebensätze, die von faktiven Nebensätzen abhingen, enthielten dagegen indikative Verbformen (DAL 1966:142f.). 
zen (vgl. 10) bzw. in sogenannten abhängigen Hauptsätzen (vgl. 11), sondern auch in Hauptsätzen auftreten (vgl. 9) und dient dem Sprecher dazu, sich von der Faktizitätsbewertung der Aussage zu distanzieren, keine Gewährleistung für ihre Wahrhaftigkeit zu übernehmen (vgl. 108, 111). AIKHENVALD (2006:135) betont zugleich: „Once this becomes the main context for the verbal form, it starts evolving into a reported evidential.“

(10) Krieger teilte mit, dass auch hierzulande die GEW die Position vertrete, den Wechsel in weiterführende Schulen anders zu gestalten. (Mannheimer Morgen, 22.12.2004)

(11) Ein Frankfurter Aktienhändler sagte, er habe den Tag mit Plaudern und Schokoladeessen verbracht. (Mannheimer Morgen, 23.12.2004)

Als Ergebnis einer solchen Entwicklung zum quotativen Evidentialitätsmarker sieht Aikhenvald die Verbform, die in Hauptsätzen des Estnischen signalisiert, dass die Information von einer anderen Person stammt. Dabei handelt es sich um ein partitives Aktivpartizip, das zuerst gemeinsam mit seinem Subjekt im Genitiv stand und mit ihm zusammen das Objekt eines übergeordneten Verbs bildete. Dann konnte nach dem übergeordneten Verb eine Subjunktion mit der Bedeutung ,dass“ eingefügt werden und das Subjekt stand im Nominativ. Schließlich entfielen sowohl das Einleitungsverb als auch die Subjunktion (vgl. 12).

(12) sai kuudla seal ühe mehe ela-vat

get.3sg.PAST hear+INF there one:GEN.SG man:GEN.SG live-PRES.PART.PARTITIVE.SG ,One learned that a man lived there“

sai kuudla (et) seal üks mees ela-vat

get.3sg.PAST hear+INF (that) there one:NOM.SG man:NOM.SG live-PRES.PART.PARTITIVE.SG

,One learned that a man lived there“

seal üks mees ela-vat

there one:NOM.SG man:NOM.SG live-PRES.PART.PARTITIVE.SG = REP.PRES

,One learned that a man lived there`

[...] In summary, the emergence of reported evidential in Estonian involved

(i) extension of complementation constructions,

(ii) reanalysis of subordinate clause as main clause, and

(iii) reinterpretation of the forms used in subordinate clauses as main clause predicates with the meaning of ,reported "information. (AIKHENWALD 2006:33, 283). 
In Grammatiken des Deutschen wird die Signalisierung der Indirektheit als der „Kernbereich“ des Konjunktivs I angesehen (FABRICIUS-HANSEN 2005:546). Allerdings gibt es auch andere Funktionsbereiche (etwa in Wunsch-, Aufforderungs- und Finalsätzen), und zwischen ihnen findet kein mit der „Finitisierung" des estnischen Partizips vergleichbarer Kategorienwechsel statt. Zudem kann der quotativ verwendete Konjunktiv I durch den Konjunktiv II oder die würde-Form ersetzt werden ${ }^{7}$, deren sonstige - insbesondere epistemisch-modale - Verwendungsbereiche noch ausgeprägter sind. Unter Umständen können in einer berichteten Rede auch Indikativformen gebraucht werden.

Auch NuYTS (2001:196, vgl. auch 208) und BuSSMANN (2002:206) bezeichnen den Konjunktiv I expressis verbis als einen Marker der quotativen Evidentialität. DE HAAN (1999:84) stellt fest, dass der deutsche Konjunktiv genauso wie der französische Konditional eine quotative Bedeutung haben kann. NUYTS (2001:313) sieht den quotativen Gebrauch des Konjunktivs als ein Beispiel für das von ihm so genannte „borrower principle“:

Thus, in grammatical and probably even in lexical expressions, there appears to be a quite systematic pattern in the double use of forms, which points in the direction of what may be called a ,borrower principle': the default marker for a qualification lower in the semantic hierarchy is borrowed by a qualification slightly higher in the hierarchy. There are numerous clear examples of this, including: the use of past tense to participate in the expression of epistemic modality, or its similar use as a marker for irreality, even independently of other epistemic epressions, common in narratives or pretend play in many languages; the use of the perfect, a marker of aspect, for expressing past time; the use of mood, supposedly a default marker for epistemic modality, for evidentiality in many languages (cf. the quotative uses of the German subjunctive) [...].

SMIRNOVA (2006:142) schlägt eine Erklärung des quotativen Gebrauchs des Konjunktivs II im Rahmen der Markiertheitstheorie von JAKOBSON (1971) vor:

Die erste binäre Unterscheidung ist diejenige zwischen dem Indikativ und dem Konjunktiv. Die zweite Unterscheidung erfolgt innerhalb der markierten Kategorie ,Konjunktiv' und wird in der deutschen Sprache als ,Konjunktiv I' und ,Konjunktiv II ‘ realisiert. Dabei lässt sich der Konjunktiv II als unmarkiertes Zeichen oder als ,Nullzeichen' auffassen. Während der Konjunktiv I explizit einen zusätzlichen Bezugspunkt ,zitierte Origo ' in seiner Bedeutung trägt und dies als positive Realisierung eines unterscheidenden Merkmals manifestiert, lässt sich der Konjunktiv II als ,Nullzeichen“ ansehen, das hinsichtlich der Realisierung dieses

7 „Beide Konjunktivkategorien [Konjunktiv I und Konjunktiv II] werden in dieser Funktion verwendet, und zwar ohne erkennbaren oder eindeutig nachweisbaren Bedeutungsunterschied.“ (FABRICIUS-HANSEN 2005:529) 
Merkmals zweideutig ist. Normalerweise tritt die konventionalisierte Bedeutung des Konjunktivs II auf, die durch die negative Ausprägung des Merkmals ,zitierte Origo" gekennzeichnet ist, also das Nicht-Vorhandensein dieses Merkmals kodiert. Die Weiterdeutung des Konjunktivs II besteht dann darin, einen unterscheidenden Aspekt für diese Form zu finden, was in der allgemeinen Annahme irgendwelcher nichterfüllter Bedingung mündet. Der Umstand, dass der Konjunktiv II als Ersatzform für den Konjunktiv I verwendet werden kann, lässt aber auch seine andere Deutung als eines Nullzeichens erkennen [...].

Legt man Aikhenvalds Terminologie zugrunde, so lassen sich wohl sowohl der Konjunktiv I als auch der Konjunktiv II und die würde-Konstruktion als evidentielle Strategien auffassen. Die Grammatikalisierung des Konjunktivs I als eines evidentiellen Ausdrucks im engen Sinne ist jedoch viel weiter fortgeschritten als bei den beiden anderen Formen. So kann der in Grammatiken des Deutschen immer noch anzutreffenden Auffassung, der zufolge der eigentliche Modus der indirekten Redewiedergabe der Konjunktiv I sei, während es sich beim Konjunktiv II und der würde-Form lediglich um „Ersatzformen“ handelt (GELHAUS 1998:165, 167), Rechnung getragen werden.

\section{Zur Semantik der würde-Form}

Da das finite Verb würde die Konjunktivmarkierung enthält, müsste die würde-Konstruktion aufgrund ihrer Form als konjunktivisch kategorisiert werden. Für zahlreiche Verwendungen (vgl. 13, 14) trifft dies auch ohne weiteres zu: Die würde-Konstruktion wird hier als eine analytische Ersatzform des entsprechenden synthetischen Konjunktivs II (Präteritum) oder als ein Konjunktiv II von werden mit Infinitiv aufgefasst. ZIFONUN (1997:1743) spricht hier von „Modalitätskontexten“, THIEROFF (1992:264) - dessen Terminus ich im Folgenden übernehme - von der „kontrafaktiven Verwendung“.

(13) Für dieses Problem sehen einige Politiker Abhilfe: Studiengebühren. Vielfach argumentieren sie, dass die Einrichtungen und die Lehre an den Unis dadurch verbessert werden könnten - vorausgesetzt, das Geld fließt an die Hochschule. Gut 70 Prozent der Studis sind gegen Gebühren. 60 Prozent würden jedoch zähneknirschend zustimmen, wenn das Geld der eigenen Alma Mater zugute käme. (Mannheimer Morgen, 8.12.2004) ${ }^{8}$

(14) Mein Vater war wirklich vor Angst ganz blaß und alt geworden. Sein Lachen klang erleichtert und doch ärgerlich. „Du Bengel“, sagte er,

8 Alle Hervorhebungen in den Belegen stammen von der Verfasserin. 
Anna Socka

„War das Ganze gespielt?“ „Leider“, sagte ich, „nicht ganz und nicht gut. Genneholm würde sagen: noch viel zu naturalistisch - und er hat recht. Schwule haben meistens recht, sie haben ein enormes Einfühlungsvermögen - aber auch nicht mehr. Immerhin. “ (BöLL 1963:184; vgl. SMIRNOVA 2006:150, 224)

In (15) bis (17) handelt es sich dagegen um sogenannte „Indirektheitskontexte“ (ZIFONUN 1997:1753), in denen die würde-Konstruktion mit dem Konjunktiv I, dem Konjunktiv II und dem Indikativ kookkurriert. ${ }^{9}$

(15) Für eine Gemeindevertretung mit 15 Abgeordneten hatten sich sowohl die „Bürger fürGroß-Rohrheim“ als auch die CDU stark gemacht. Während BfGR-Sprecher Walter Öhlenschläger im Verlaufe der Debatte darauf beharrte, beantragte die Union eine Sitzungsunterbrechung. Daraus kehrte Fraktionschef Georg Menger mit der Nachricht zurück, dass die Christdemokraten mit der SPD für die 19 Sitze stimmen würden, um die Schrumpfkur noch zu retten. (Mannheimer Morgen, 9.12.2004)

(16) Das auch unter dem Namen Norwalk bekannte Noro-Virus werde über Fäkalien und Erbrochenes übertragen, hieß es. Die Symptome würden normalerweise spätestens nach 42 Stunden wieder abklingen, sagte Sprecherin Glasmacher. (Mannheimer Morgen, 29.12.2004)

(17) Vor allem der Kommunikationsgedanke stehe im Vordergrund, betonte auch Jeanette Klee. Schließlich gebe es einige ältere Menschen, die zum Teil auch nicht mehr in der Lage seien, für sich selbst zu kochen, oder Gesellschaft während des Essens bevorzugen würden. (Mannheimer Morgen, 17.12.2004)

Die Verwendbarkeit der würde-Konstruktion in indikativischen Kontexten wie (18)-(20) spricht dagegen für ihre Behandlung als indikativisch. In (18) werden Überzeugungen der Sektenanhänger in einem Komplementsatz wiedergegeben. In (19) handelt es sich um die sogenannte erlebte Rede, d.h. um mehrere Hauptsätze im Indikativ Präteritum, in denen Gedanken einer Romanfigur wiedergegeben werden. In (20) erscheint die würde-Konstruktion ebenfalls in einem indikativisch-präteritalen Kontext und bezeichnet Zukünftiges relativ zu

9 In (15) ist es ein Komplementsatz, in (16) folgt die Redeeinleitung (,,sagte Sprecherin Glasmacher") der Wiedergabe nach. In (17) ist ein Hinweis auf die Sprecherin im Vortext enthalten, ansonsten ist die Indirektheit nur durch den Verbmodus (im Defaultfall den Konjunktiv Präsens) markiert. 
einem vergangenen Zeitpunkt, es handelt sich jedoch nicht um eine Wiedergabe von Äußerungen oder Gedanken.

(18) Die Sekten-Anhänger glaubten, dass die Jahrtausend-Wende den Weltuntergang mit sich bringen würde. (Zit. nach KOTIN 2003:229)

(19) Deshalb wollte er sich schon bei Anbruch der Dämmerung, ehe noch die Tore geschlossen wurden, einschleichen und im Schutz der eigenen Geruchlosigkeit [...] in irgendeinem Winkel des Hauses verbergen. Später dann, wenn alles schlief, würde er, vom Kompass seiner Nase durch die Dunkelheit geführt, zur Kammer seines Schatzes hinaufsteigen. Er würde ihn an Ort und Stelle im fettgetränkten Tuch verarbeiten. Nur Haar und Kleider würde er wie gewöhnlich mitnehmen, da diese Teile direkt in Weingeist ausgewaschen werden konnten, was sich bequemer in der Werkstatt machen ließ. (SÜSKIND 1985:267f.)

(20) Vielleicht war der Abend damals genauso magisch, vor hundert Jahren, als sich die Häuptlinge und Großmänner der Herero an der Viehtränke Onguera zur Beratung versammelten. Am Vortag, es war der 11. August 1904, hatte die Entscheidungsschlacht begonnen, die Herero hatten sich wacker geschlagen, aber die zahlenmäßig weit überlegene deutsche Kolonialtruppe mit ihren Krupp-Geschützen würde sie besiegen. Es war nur eine Frage der Zeit. (GRILL 2004:10)

Belege, in denen die Form Zukunftsbezug hat $(13,15,18-20)$ sprechen dafür, dass es sich um eine Futurform handelt. Die Belege (14), (16), (17) sprechen jedoch dagegen: Hier fungiert die Form eher als ein analytischer Ersatz des Konjunktiv Präteritum des jeweiligen Verbs (FABRICIUS-HANSEN 1998:140). Während die meisten Grammatiken des Deutschen (z.B. GelHAus 1998:133) die Konstruktion als „ein präteritales konjunktivisches Pendant des Futurs“ (FABRICIUS-HANSEN 1998:141) beschreiben, wird sie in WeINRICH (1993) und ZIFONUN (1997) als eine analytische Form des Konjunktiv II aufgefasst. THIEROFF (1992) stellt dagegen besonders Fälle wie (19) und (20) heraus und behandelt die Konstruktion als ein modusneutrales Futur Präteritum. Für jede dieser Auffassungen lassen sich Belege und theoretische Argumente anführen, die sie bestätigen, wie auch andere, die im Hinblick auf sie Probleme bereiten (vgl. dazu FABRICIUS-HANSEN 1998 und SOCKA 2006). Keine von ihnen wird der komplexen Semantik der würde-Konstruktion gerecht.

Das Ziel von FABRICIUS-HANSEN (2000) ist eine kompositionale Bedeutungsbeschreibung der würde-Konstruktion, d.h. eine solche, in der die Bedeutung 
eines zusammengesetzten Ausdrucks sich aus den Bedeutungen seiner Bestandteile und der Art der Zusammensetzung ergibt. Die Bedeutung der würdeKonstruktion wird aus dem Zusammenspiel der Bedeutungen des finiten präsentischen werden mit Infinitiv einerseits und des präteritalen Konjunktivs andererseits abgeleitet. Die Bedeutung der Konstruktion werden+Infinitiv wird als „Non-Realis“ charakterisiert. Damit ist gemeint, dass mit einer Aussage der Form „wird (p)“ der Sprecher entweder behauptet, dass p mit Bezug auf eine zeitliche Fortsetzung der aktuellen Faktenwelt wahr ist (temporale Lesart) oder dass $p$ relativ zu einer epistemischen Erweiterung seines Wissens zur Faktenwelt gehört (epistemisch-modale Lesart, vgl. FABRICIUS-HANSEN 2000:87f.). Die Disambiguierung zwischen den beiden Lesarten erfolgt durch den Kontext sowie aufgrund semantischer Eigenschaften des Vollverbs. Mit dieser Auffassung sieht sich Fabricius-Hansen in einer Beschreibungstradition, nach welcher der für beide Lesarten gemeinsame Bedeutungskern im (vorläufigen) Fehlen einer direkten Evidenz für die behauptete Proposition besteht. Wenn dagegen SMIRNOVA (2006) werden+Infinitiv als Ausdruck für das Vorhandensein indirekter Evidenzen betrachtet, klingt dies wie eine etwas positivere Formulierung desselben Gedankens. FABRICIUS-HANSEN (2000:87) betont allerdings, dass in ihrem Ansatz ,nicht so sehr die Dimension der Evidenz wie die des Realen, des Faktischen im Zentrum [steht]". Grundlegend für die Theorie von Fabricius-Hansen ist ferner die Annahme, dass der präteritale Konjunktiv im heutigen Deutsch zwei Funktionen hat, d.h. er signalisiert entweder Indirektheit (wie in 15-19) oder Kontrafaktivität (wie in 13-14). Beiden Funktionen ist gemeinsam, dass die Konjunktiv-Präteritum-Markierung eine Verschiebung bewirkt. Bei Indirektheit ist es eine Verschiebung der SprecherOrigo: Der aktuelle Sprecher mit seiner epistemischen Perspektive wird durch eine andere Person ersetzt. Bei Kontrafaktivität handelt es sich um eine Verschiebung der Welt-Origo: Es muss eine Welt berücksichtigt werden, die von der, wirklichen" Welt abweicht. Keines von beiden liegt allerdings in Fällen wie (20) vor: Hier handele es sich um eine „sekundäre Verwendung“ (FABRICIUS-HANSEN 2000:95) der Konstruktion.

Auch SMIRNOva (2006:171) geht von einer komplexen Bedeutungsstruktur der Konstruktion würde+Infinitiv aus, die sie wie folgt darstellt: 
Abb. 1:

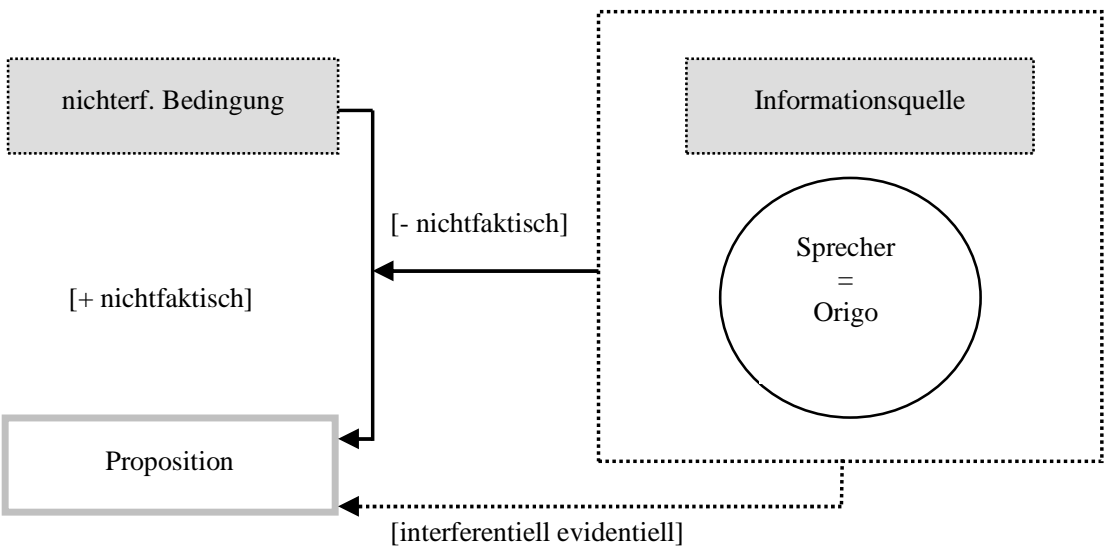

Die Struktur enthält zwei Relationen: (i) die durch werden+Infinitiv ausgedrückte inferentielle Relation, ,die die Proposition als Folgerung aus den dem Sprecher vorliegenden Informationen (Prämissen) kennzeichnet“ und (ii) eine „Relation, die die Nichtfaktizität der Proposition aufgrund der nichterfüllten Bedingung präsentiert“ (SMIRNOVA 2006:172). Die letztere Relation macht die Bedeutung des Konjunktivs II aus, bei dem es sich folglich um einen grammatikalisierten deiktischen Modalitätsmarker handelt. ${ }^{10}$ Die werden-Relation ist in die konjunktivische Relation eingebettet. Abhängig vom Verwendungskontext können nun unterschiedliche „Spezifizierungen deiktisch determinierter Relationen zu unterschiedlichen Bedeutungen führen“ (SMIRNOVA 2006:171). So ordnen übergeordnete Verben wie glauben, vermuten, wissen im Präteritum (vgl. 18) „den Inhalt der Proposition der epistemischen Erweiterung der faktischen Welt zu [...] und [entziehen] somit die Aussage der festen Bindung an eine sprachlich formulierbare nichterfüllte Bedingung" (SMIRNOVA 2006:138154). Die konjunktivische Interpretation der Konstruktion würde+Infinitiv

10 Smirnova folgt hier Diewald (1999:186), der zufolge der Konjunktiv II einer Proposition aufgrund einer nichterfüllten Bedingung (die nicht unbedingt explizit formuliert, doch grundsätzlich sprachlich formulierbar ist) einen negativen Faktizitätswert zuweist. Die Konjunktiv-II-Semantik enthält also einerseits einen phorischen Verweis auf die nichterfüllte Bedingung, andererseits ,bringt er zum Ausdruck, dass dieser Zusammenhang zwischen der nicht-erfüllten Bedingung und dem Faktizitätswert vom Sprecher hergestellt, also von der Sprecherperspektive aus gesetzt wird“"(DIEWALD 1999:189). 
wird blockiert, vielmehr ist sie als ,modusneutrale präteritale Entsprechung von werden+Infinitiv" zu verstehen. Analog - als Versprachlichung von Schlussfolgerungen „referierter Personen“ - sieht Smirnova den Gebrauch der würdeKonstruktion in der erlebten Rede. Zu Beispielen wie (20) äußert sie sich nicht, es steht jedoch zu vermuten, dass derartige Verwendungen sich relativ leicht in ihr Modell integrieren lassen, da sie zwar vom ,aktuellen Sprecher als [...] Zwischeninstanz“ oder von „referierten Personen“ spricht, aber keine Origo-Verschiebung oder Versetzungsdeixis postuliert. ${ }^{11}$

In ihrer zweiten (kontrafaktiven) Lesart fungiert die würde-Konstruktion dagegen als ein analytischer Konjunktiv II. Seinen Hauptverwendungskontext bildet der Hauptsatz einer inhaltlichen konditionalen Satzperiode (vgl. 13). ${ }^{12}$ In der Bedeutungsstruktur der würde-Konstruktion tritt hier die konjunktivische Relation zwischen der im Nebensatz verbalisierten nichterfüllten Bedingung und der im Hauptsatz ausgedrückten Proposition in den Vordergrund, während außersprachliche Evidenzen als Ausgangspunkt der inferentiellen werden-Relation ausgeblendet werden und folglich die ganze Relation eliminiert wird.

Als eine dritte Lesart unterscheidet SMIRNOVA (2006:148) den „Konjunktiv II von werden+Infinitiv“, bei dem es sich semantisch um die „,durch die Konjunktivform abgeschwächte evidentielle Bedeutung der Konstruktion werden+Infinitiv" handelt (SMIRNOVA 2006:155). Wie ihr Beispiel (14) zeigt, ist sie weder auf präteritale noch auf Indirektheitskontexte beschränkt. SMIRNOVA (2006:152f.) ist ferner der Meinung, dass die inferentielle Bedeutung solcher würde-Sätze (Schlussfolgerung aus außersprachlichen Evidenzen) auch durch explizit konditionale Paraphrasen nicht tangiert wird. Der Konjunktiv hat eine abschwächende Funktion, d.h. seine Bedeutung wird als, phorisch verankerte bedingte Faktizität' reinterpretiert. Auch in diesem Punkt nähert sich die Auf-

11 Dies hängt möglicherweise auch damit zusammen, dass SMIRNOVA (2006:184) nicht zwischen den beiden narratologischen Instanzen Autor und (Ich-)Erzähler unterscheidet, sondern mit Bezug auf die Ansichten eines Clowns feststellt: „Es werden eigene Gedanken des Autors wiedergegeben, der zugleich als Protagonist auftritt."

12 Unter einer inhaltlichen Verknüpfung wird dabei eine konditionale Relation verstanden, in der die beiden Sätze ,semantisch kausal verbunden [sind]: die Protasis wird inhaltlich als Grund für die Apodosis als Folge angesehen" (SMIRNOVA 2006:198). Ferner sei diese Lesart für konditionale Nebensätze sowie für Hauptsätze von konzessiven, konsekutiven und komparativen Satzgefügen charakteristisch, „wobei der Gebrauch von konjunktivischen verbalen Formen nicht nur semantisch motiviert ist, sondern vielmehr nach syntaktischen Regeln (als Subjunktiv) erfolgt" (SMIRNOVA 2006:183). 
fassung Smirnovas derjenigen von FABRICIUS-HANSEN (2000:90), selbst wenn Fabricius-Hansen diese Lesart der kontrafaktiven zuschlägt:

Der präsentischen und der präterital-konjunktivischen Konstruktion ist gemeinsam, dass sie das beschriebene Geschehen in einer aus der Sicht des aktuellen Sprechers nicht wirklichen Welt ansiedeln, die jedoch im Präsens-Fall mit der bis zum relevanten Jetzt hinreichenden Wirklichkeit verträglich sein muss [...]. Im irrealen würde-Fall, um den es hier geht, handelt es sich der Funktion des irrealen Konjunktiv entsprechend um eine Welt, die in bestimmter Weise von der wirklichen Welt bzw. einer erwartbaren Fortsetzung davon abweicht [...]

Anders als bei Smirnova sind allerdings in dieser Auffassung die Grundbedeutungen der Fügung werden+Infinitiv einerseits und des Konjunktivs Präteritum andererseits beide modal und folglich im Wesentlichen gleich: Es sind zwei verschiedene Grade der Abweichung von der aktuellen Faktenwelt. Der Konjunktiv Präteritum stellt die Sachverhalte als nur hypothetisch möglich dar, während beim Gebrauch der werden-Konstruktion die Sachverhalte als wahrscheinlich erscheinen: Sie werden geplant, erwartet, befürchtet etc. Ohne zugunsten dieser Auffassung zu argumentieren, sei hier nur darauf hingewiesen, dass in allen von SMIRNOvA (2006:148-153, 224f.) angeführten Belegen ${ }^{13}$ durch den Kontext ,[...] nicht realisierte Umstände bereitgestellt [werden], auf deren Hintergrund ein [mithilfe des würde-Satzes ausgedrückter] nicht-faktischer Sachverhalt zu sehen ist“" (ZIFONUN 1997:1748; DIEWALD 1999:186). Diese nicht realisierten Umstände lassen sich auch relativ mühelos in einem Bedingungssatz paraphrasieren. In Smirnovas Beispielen betreffen diese Umstände zudem konkrete, singuläre Personen, weswegen konjunktivische Aussagen über sie (z. B. Genneholm würde sagen ... in Beispiel 14) in der Tat als Schlussfolgerungen aus der Kenntnis dieser Personen, d.h. als inferentiell, interpretiert werden können. Da sie aber zugleich an die nicht-realisierten Um-

13 Ausnahmen bilden ihre Beispiele (42): ... eine ständige Gereiztheit, weil er [Karl Emonds] sich ausrechnen konnte, dass er nie eine für ihn angemessene Wohnung würde bezahlen können (BöLL 1963; zit. nach SMIRNOVA 2006:149), und (52) Wir würden heute sagen, er versuchte, das Wesen der Energie realontologisch auszudeuten (BАMм 1977:225). Beim ersten Beispiel handelt es sich um die erste (rein inferentielle) Bedeutungsvariante, beim zweiten um eine Art Höflichkeitskonjunktiv, mit der pragmatischen Funktion ,über eine Abschwächung des Faktizitätsanspruches eine Abmilderung der mit der Äußerung verbundenen interaktionsbezogenen Verbindlichkeiten [zu erreichen]“ (ZIFONUN 1997:1753). 
stände gebunden sind ${ }^{14}$, fasse ich diese dritte Lesart als eine Variante der zweiten (kontrafaktiven) auf.

Einen radikal entgegengesetzten Ansatz verfolgt neuerdings WeLKE (2005:448), nach dessen Ansicht es sich bei der Fügung werden+Infinitiv um ein Futur handelt, d.h. die Bedeutung ,Zukunft ' ist für sie in diachroner Sicht primär und für den heutigen Gebrauch typisch. Hat die Fügung eine epistemisch-modale Interpretation, so kommt sie lediglich aufgrund „rekonstruierbare[r] Implikaturen" zustande. Mit Bezug auf die würde-Konstruktion betont Welke, dass ihre verschiedenen Bedeutungsvarianten voneinander geschichtlich abgeleitet wurden und folglich keine durchgehende Kompositionalität, sondern höchstens „Familienähnlichkeiten“ aufweisen können. Am Anfang des Ableitungsprozesses stand die sogenannte archetypische Bedeutung der würde-Konstruktion, zukünftig relativ zu einer vergangenen Originaläußerungszeit', die ursprünglich in abhängigen Nebensätzen nach präteritalen Verben des Meinens und Sagens vorkam (vgl. 18). Davon führt ein „Entwicklungspfad“" zur kontrafaktiven zukunftsbezogenen Bedeutungsvariante, die sich nach WeLKe (2005:476) als „Konjunktiv Präteritum zu dem Indikativ Futur“ analysieren lässt (vgl. 13). In einem weiteren Entwicklungsschritt wurde davon die gegenwartsbezogene Variante ,analytischer Konjunktiv“ abgeleitet (vgl. 14 sowie Konditionalgefüge wie Diese Aussage wäre überzeugender, würden im Erinnerungsvermögen des Zeugen Schulz nicht arge Lücken klaffen, zit. in ZIFONUN 1997:1744). Ein anderer Entwicklungspfad umfasst den Bedeutungswandel der würde-Konstruktion in Indirektheitskontexten. Aus der archetypischen Bedeutungsvariante, bei der die neue Origo mithilfe eines regierenden Verbs des Sagens oder des Meinens in das Präteritum gesetzt wird, entsteht zuerst eine weitere, die Zukünftiges relativ zu einem Zeitpunkt ausdrückt, an dem eine im präteritalen Erzähltext erwähnte Figur denkt, plant, fühlt etc. (vgl. 19). WELKE (2005:469f.) bezeichnet sie deshalb als ,deixisverschoben“ und analysiert sie als „Indikativ Futur Präteritum“. In einem weiteren Entwicklungsschritt entfällt der Bezug auf eine neue Origo, deswegen spricht

14 Ferner scheinen mir diese nicht realisierten Umstände (bzw. unerfüllten Bedingungen) auch nicht immer so „nebensächlich“ zu sein, wie sie SMIRNOVA (2006: 152) sehen möchte, vor allem nicht in ihren Belegen (49) Sabeth würde es natürlich anders taufen, aber ich weiß nicht wie und (50) Sabeth würde sagen: wie Smaragd! (SmiRnOva 2006:150). Beide Sätze erscheinen im Roman Homo faber im Kontext der Gedanken Walter Fabers nach dem Tod von Sabeth, weswegen man hier auch schwierig von einer lediglich ,abgeschwächten Faktizität“ sprechen kann. 
WELKE (2005:472, s. Beispiel 20) vom „nicht-deixisverschobenen“ Indikativ Futur Präteritum. In SOCKA (2008) habe ich auf einige Schwierigkeiten hingewiesen, die der Ansatz von Welke bei der Analyse von Korpusbeispielen bereitet und die folglich eher für die Auffassung von FABRICIUS-HANSEN (2000) sprechen. Im Folgenden soll auf Möglichkeiten eingegangen werden, die eine Analyse der würde-Konstruktion als inferentiellen Ausdruck im Sinne von Smirnova eröffnet.

\section{Korpusbelege}

Die in diesem Abschnitt zu besprechenden Belege wurden der Tageszeitung Mannheimer Morgen entnommen. ${ }^{15}$ Es handelt sich sämtlich um Meldungen, in denen über Kommunikationsakte bezüglich verschiedener politischer, wirtschaftlicher oder sozialer Maßnahmen berichtet wird; der Kontext motiviert also die quotative Interpretation der würde-Formen. Darüber hinaus sind sie aber oft zwischen der evidentiellen und der kontrafaktiven Lesart ambig. 10 muttersprachliche Informanten mit philologischem Hintergrund wurden befragt, ob die würde-Form bereits für die Originaläußerung anzunehmen ist. Eine positive Antwort wurde als eine Entscheidung für die kontrafaktive Lesart aufgefasst.

Der Ansatz von Smirnova ermöglicht eine einleuchtende Erklärung von Beispiel (21), bei dem sich 8 von 10 Informanten für geben wird in der Originaläußerung (also den Voraussagen der Fans o. Ä.) aussprachen, obwohl sie es zugleich „komisch“ fanden, da „man das Ergebnis eines Fußballspiels schlecht voraussagen kann“. Die Voraussage lässt sich aber ohne weiteres als Schlussfolgerung aus Prämissen auffassen, die nachfolgend sogar detailliert ausgebreitet werden.

(21) Mit der Brechstange zum 1:1 FUSSBALL: Gladbach trotzt Leverkusen verdient einen Punkt ab. Aus Mönchengladbach berichtet unser Mitarbeiter Ludwig Ricke

Irgendwie war es ja klar, dass es im Duell zwischen Borussia Mönchengladbach und Bayer Leverkusen keinen Sieger geben würde. Die Fohlen gewannen seit 15 Jahren zu Hause gegen die Werkself nicht mehr und Bayer konnte nach einem Champions-League-Spiel

15 Herangezogen wurden die im Korpus der geschriebenen Sprache des Instituts für Deutsche Sprache in Mannheim online recherchierbaren Ausgaben der Zeitung vom Dezember 2004. 
Anna Socka

in der Bundesliga bislang nie siegen. (Mannheimer Morgen, 13.12.2004)

Ähnlich lässt sich die würde-Form in indirekten Fragesätzen erklären, die von den Informanten auch mehrheitlich auf indikativisches werden in der Originaläußerung zurückgeführt wurden: bei Beleg (22) waren alle Informanten dieser Meinung, bei Beleg (23) 7 von 10 Befragten. Behandelt man (indirekte) Fragesätze als Mengen von wahren Satzantworten auf sie (BÄUERLE / ZIMMERMANN 1991:344), so besteht die Einstellung der Fragenden (Christa Ludwig in 22, der Schulkinder in 23) in der Suche nach Evidenzen für die einzelnen Elemente dieser Menge.

(22) Peter Rehkop, der Retter der Premiere der Mozartwoche, stellte sich mit zwei Arien aus Mozarts „Ascanio“ vor. Einen Countertenor hatte Christa Ludwig noch nie unter ihren Fittichen und so war sie sehr zögerlich, was sie ihm würde vermitteln können. Aber sie konnte: Rehkop sang sicherer in der Tongebung, dramatischer im Ausdruck, volltönender im Timbre. (Mannheimer Morgen, 10.12.2004)

(23) Die Erlaubnis der Gemeinde Neulußheim musste eingeholt werden, mit Unterstützung von Walter Merz vom Obst- und Gartenbauverein und engagierten Eltern aus der Markus-Schule wurde ein junger Apfelbaum gekauft, im Schulhof ein Loch ausgehoben und Muttererde besorgt. Am 24. November war es endlich soweit. Alle Grundschüler versammelten sich im Schulhof und bildeten einen großen Kreis um den Apfelbaum, der bereits an seinem neuen Platz stand, und warteten in feierlicher Spannung, was nun geschehen würde. (Mannheimer Morgen, 1.12.2004)

Die Informanten machten ihre Entscheidung generell davon abhängig, ob die Maßnahmen für die Zukunft bereits beschlossen, eingeplant sind, oder ob sie eher nur hypothetisch erwogen werden. So waren bei Beleg (24) vier von zehn Personen für nicht übernehmen wird in der Originaläußerung, während sechs für nicht übernehmen würde plädierten, mit der Begründung, es handle sich um ein Projekt, das noch gebilligt werden [muss]. Die (noch) nicht erfüllte Bedingung lässt sich leicht in einem konditionalen Nebensatz formulieren, 
etwa: Wenn der Stadtvorstand und die städtischen Gremien zustimmten... Dies spricht für die kontrafaktive Lesart. ${ }^{16}$

(24) Stadt und Förderverein erörtern neue Finanzlösung / Ganz neue Entwicklungen beim Bliesbad: Der Vorstand des Fördervereins hat gestern Sportdezernent Wolfgang van Vliet einen Vorschlag unterbreitet, mit dem dieser nach eigenen Aussagen ,sehr gut leben könnte “. [...]

Mit diesen Regelungen werden Jahreseinnahmen von rund 37000 Euro erwartet. Der Förderverein, der das Bad seit zehn Jahren betreibt, denkt aber auch daran, eine Jahres-Familienkarte für 70 Euro anzubieten und den Mitgliedern besondere Konditionen zu gewähren (Infos unter Tel. 0621/68 30 85). Reimann: „Wir wollen weiterhin ein soziales Bad bleiben."

Nicht übernehmen würde der Förderverein die Bewirtschaftung des Parkplatzes. Die Bedingungen wurden als , unannehmbar" bezeichnet. Van Vliet will dem Stadtvorstand am Dienstag den Vorschlag unterbreiten. Wenn dieser zustimmt, muss diese Regelung aber noch von den stadträtlichen Gremien gebilligt werden. (Mannheimer Morgen, 17.12.2004)

In Beleg (25) wird dagegen die Äußerung eines Lokalpolitikers wiedergegeben, der kürzlich eine Verkleinerung der gemeindlichen Gremien mitbeantragt hat. Aus dem Kontext geht hervor, dass die Verkleinerung bereits beschlossen (abgesegnet) wurde, der Politiker äußert sich über die zu erwartenden positiven Folgen dieser Maßnahme. Deswegen vermuten sechs Informanten, dass der Originalsprecher hier das inferentielle werden+Infinitiv gebraucht hat. Jedoch besteht keine Einigkeit: Drei Personen konnten sich nicht zwischen den beiden Möglichkeiten entscheiden, eine war für die würde-Form in der Originaläußerung.

(25) Den Antrag zur Verkleinerung der gemeindlichen Gremien hatten die stärksten Fraktionen, CDU und SPD, gemeinsam gestellt und während der jüngsten Gemeindevertretung mit der erforderlichen Zweidrittel-Mehrheit abgesegnet. BBF und FLB stimmten dagegen. Bereits im Sommer hatte die CDU-Fraktion über die Parlamentsverkleinerung diskutiert, erklärte Fraktionschef Hans Michael Platz.

16 Die Entscheidung für die kontrafaktive Lesart wurde wahrscheinlich auch dadurch begünstigt, dass hier die Konjunktiv-II-Form gebraucht wird, obwohl der Konjunktiv I übernehmen werde ebenfalls eindeutig gewesen wäre. 
Anna Socka

Die zahlenmäßig stärkste Fraktion wollte den Vorstoß nicht allein machen, sondern holte sich mit der SPD die zweitgrößte Gruppe ins Boot. So entstand der gemeinsame Antrag.

Bei der nächsten Wahl werden nur noch 23 Plätze in der Gemeindevertretung zu vergeben sein. Im Moment gibt es dort 31 Sitze. Die Ortsbeiräte schrumpfen von neun auf sieben Mitglieder. Die Zahl der Beigeordneten im Gemeindevorstand sinkt von sieben auf fünf.

Der Bürgerwille komme auch in einem kleineren Parlament zum Ausdruck, meinte Fraktionschef Platz. Wenn weniger Leute am Tisch sitzen, gebe es einen „schnelleren Entscheidungsfluss“. Die Gemeindevertretung arbeite effizienter, und das sei der Hauptgrund für die Verkleinerung, betonte Platz. Die kleinen Parteien sieht er nicht benachteiligt. Im Gegenteil, diese würden mit weniger Stimmen mehr Sitze erhalten. „Leidtragende sind die großen Parteien, die mehr Federn lassen müssen", sagte Platz. (Mannheimer Morgen, 17.12.2004)

Bei Beleg (26) plädierte die Hälfte der Informanten für werden+Infinitiv in der Originaläußerung (feiern wird). Die andere Hälfte vertrat aber die Meinung, dass der Originalsprecher auch schon die würde-Form benutzen könnte, um anzudeuten, dass er nicht sicher ist, ob die angeschlagene Firma an ihrem 125. Jahrestag noch existieren wird. Diese Unsicherheit lässt sich als eine Abschwächung der evidentiellen Bedeutung durch die Konjunktiv-II-Markierung deuten. Es handelt sich also um Smirnovas dritte Lesart (,Konjunktiv II von werden+Infinitiv").

(26) Der traditionsreiche Pumpenhersteller, der im kommenden Jahr das 125-jährige Bestehen feiern würde, befinde sich in großen und dauerhaften Schwierigkeiten, sagte Plingen. Die Wettbewerbslage am Markt sei „sehr scharf“. (Mannheimer Morgen, 17.12.2004)

\section{Abschließende Bemerkungen}

Die seit mehreren Jahren intensiv diskutierte Frage einer adäquaten Bedeutungsbeschreibung der Konstruktion würde+Infinitiv (z. B. THIEROFF 1992; FABRICIUS-HANSEN 1998, 2000; WELKE 2004) erhielt durch die Idee von SMIRNOVA (2006), bei werden+Inifintiv handele es sich um einen Ausdruck der inferentiellen Evidentialität, neue Impulse. Begreift man die würde-Konstruktion zudem, soweit sie in Indirektheitskontexten erscheint, als eine evidentiell-quotative Strategie, so lässt sich die oben genannte Frage neu formu- 
lieren: Unter welchen kontextuellen Bedingungen wird jeweils die modale, die inferentielle und die quotative Bedeutungskomponente aktiviert bzw. blockiert? Wie bedingen sie sich gegenseitig? Die Funktion der würde-Konstruktion als quotative Strategie kann als ein möglicher Auslöser für die Ausschaltung der kontrafaktiven und die Aktivierung der rein inferentiellen Interpretation aufgefasst werden. Als quotative Strategie wäre dann auch der Gebrauch der Konstruktion in erlebter Rede zu betrachten, in der - wie in Beleg (19) - Gedanken eines im indikativischen Text erwähnten „Bewußtseinsträgers“ (vgl. RoNCADOR 1988) wiedergegeben werden. Das Vorliegen einer quotativen Strategie ist jedoch keine hinreichende Bedingung für die rein inferentielle Interpretation. Vielmehr kann - wie Beleg (24) zeigt - auch das quotative würde+Infinitiv eine kontrafaktive Interpretation haben, die dann allerdings bereits für die wiedergegebene Originaläußerung anzunehmen ist. Liegt indessen keine quotative Strategie vor, so erhält die würde-Konstruktion in der Regel die kontrafaktive Interpretation. Dies soll das untenstehende Schema verdeutlichen, in dem ich unter dem Stichwort, kontrafaktiv' Smirnovas zweite und dritte Lesart zusammenfasse und der ersten (,rein inferentiellen') gegenüberstelle. Unter der horizontalen Linie erscheinen in der Abbildung zwei weitere Verwendungsweisen, die Smirnova außer Acht lässt. Zum einen hat würde mit Infinitiv in Belegen wie (20) inferentielle Bedeutung ${ }^{17}$, ohne dass zugleich eine quotative Strategie vorläge. Zum anderen lässt sich der quotativ gebrauchten würde-Konstruktion in Belegen wie (16) wohl keine inferentielle Interpretation zuordnen. Vielmehr handelt es sich lediglich um einen Ersatz für uneindeutige Konjunktiv-I- bzw. ungebräuchliche Konjunktiv-II-Formen. In beiden Fällen spricht FABRICIUS-HANSEN (2000:93) von einem „Funktionsausgleich“.

17 Diese Bedeutung wird verstärkt durch den Satz Es war nur eine Frage der Zeit. Kommentare dieser Art sind für solche Belege durchaus typisch, was wohl damit zusammenhängt, dass oft über Ereignisse berichtet wird, für deren zukünftiges Eintreten zu dem vergangenen Bezugspunkt bereits Evidenzen vorgelegen haben (in Beleg 20 ist es etwa die zahlenmäßige und wehrtechnische Überlegenheit der Kolonialtruppe). 
Abb. 2:

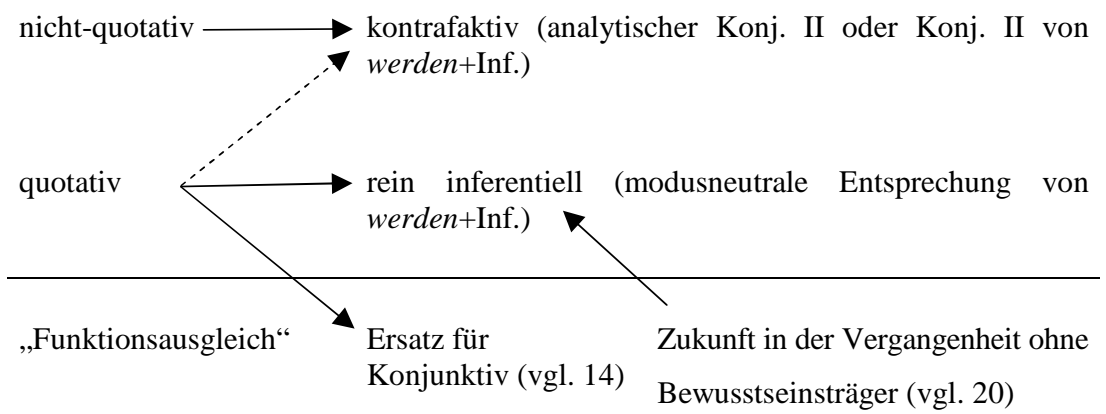

Eine wichtige Rolle scheint ferner die Semantik von redeeinleitenden (nicht unbedingt syntaktisch übergeordneten) Verben zu spielen. Dieser und wahrscheinlich noch andere Faktoren müssen späteren Untersuchungen vorbehalten bleiben.

\section{Literatur}

Aikhenvald, Alexandra Y. (2006): Evidentiality. Oxford.

ANDERSON, LLOYD B. (1986): Evidentials, paths of change and mental maps: Typologically regular assymetries. In: CHAFE / NICHOLS, 273-312.

AuWERA, JOHAN VAN DER / Plungian, VLADIMIR (1998): On modality's semantic map. In: Linguistic Typology 2:79-124.

Bäuerle, Rainer / Zimmermann, Thomas Ede (1991): Fragesätze. In: Stechow, ARNIM VON / Wunderlich, DiETER (eds.): Semantik. Ein internationales Handbuch zeitgenössischer Forschung. Berlin/New York (=Handbücher zur Sprach- und Kommunikationswissenschaft 6), 333-248.

BAMM, PETER (1977): Ex ovo. München.

BöLL, HEINRICH (1963): Ansichten eines Clowns. Köln/Berlin.

BuSSMANN, HADUMOD (1983 / $\left.{ }^{3} 2002\right)$ : Lexikon der Sprachwissenschaft. Stuttgart.

CHAFE, WALLACE (1986): Evidentiality in English conversation and academic writing. In: CHAFE / NiCHOLS, 261-272.

Chafe, Wallace / Nichols, Johanna (eds.) (1986): Evidentiality: The linguistic coding of epistemology. Norwood.

DAL, INGERID (1966): Kurze deutsche Syntax auf historischer Grundlage. Tübingen. 
Die Konstruktion würde+Infinitiv als evidentieller Ausdruck des Deutschen

Dendale, PATRICK / TASMOWSKi, Liliane (2001): Introduction: Evidentiality and related notions. In: Journal of Pragmatics 33:339-348.

Diewald, Gabriele (1999): Die Modalverben im Deutschen. Grammatikalisierung und Polyfunktionalität. Tübingen (=Reihe Germanistische Linguistik 208).

- (2004): Faktizität und Evidentialität: Semantische Differenzierungen bei den Modalund Modalitätsverben. In: LeIRBUKT, ODDLEIF (ed.): Tempus/Temporalität und Modus/Modalität im Sprachvergleich. Tübingen (=Eurogermanistik 18), 231-256.

- (2005): Werden \& Infinitiv - Versuch einer Zwischenbilanz nebst Ausblick. In: Deutsch als Fremdsprache 42:23-32.

Fabricius-Hansen, CATHRINE (1998): Über den Platz des würde-Gefüges im TempusModus-System des gegenwärtigen Deutsch. In: PORS, HARALD / JAKOBSEN, LisBETH Falser / StubkJaer, FlenNig Talbo (eds.): Sprachgermanistik in Skandinavien III. Akten des IV. Nordischen Germanisten-Treffens auf Schloß Sandbjerg 5.-8. Juni 1996. Århus, 135-158.

- (2000): Die Geheimnisse der deutschen würde-Konstruktion. In: THIEROFF, Rolf / Funrkop, Nanna / Teuber, Oliver (eds.): Deutsche Grammatik in Theorie und Praxis. Tübingen, 83-96.

- (2005): Das Verb. In: DudENREDAKTION (ed.): Duden. Die Grammatik. Unentbehrlich für richtiges Deutsch. Mannheim, 395-572.

FALler, Martina (2007): The Cuzco Quechua Reportive evidential and rhetorical relations. In: AUSTIN, PETER K. / SIMPSON ANDREW (eds.): Endangered languages. Linguistische Berichte Sonderheft 14:223-251.

Fritz, Thomas A. (2000): Wahr-Sagen: Futur, Modalität und Sprecherbezug im Deutschen. Hamburg (=Beiträge zur germanistischen Sprachwissenschaft 18).

Gelhaus, Hermann (1998): Die Wortarten. In: Dudenredaktion (ed.): Duden. Grammatik der deutschen Gegenwartssprache. Mannheim, 85-407.

GRILl, BARTOLOMÄUS (2004): Aufräumen, aufhängen, niederknallen! In: Die Zeit, 5.08.2004:19.

HAAN, FERDINAND DE (1999): Evidentiality and epistemic modality. Setting boundaries. In: Southwest Journal of Lingustics 18:83-101.

- (2001): The place of inference within the evidential system. In: International Journal of American Linguistics 67:193-219.

- (2005): Encoding speaker perspective: Evidentials. In: FRAJZYNGIER, ZYGMUNT / Hodges, ADAm / Rood, DAvid S. (eds.): Linguistic Diversity and Language Theories. Amsterdam (=Studies in Language Companion Series 72), 379-397.

HASSLER, GERDA (2002): Evidentiality and reported speech in Romance languages. In: Güldemann, Tom / Roncador, MANFred von (eds.): Reported Speech. A Meeting Ground for Different Linguistic Domains. Amsterdam, 143-172.

Helbig, Gerhard / Buscha, JoAChIM (2001): Deutsche Grammatik. Ein Handbuch für den Ausländerunterricht. Berlin.

Helbig, Gerhard / Helbig, Agnes (1990): Lexikon deutscher Modalwörter. Leipzig. 
JAKOBSON, Roman (1939): Signe zéro. In: JAKOBSON, RomAN (1971): Selected Writings. Vol. 2: Word and Language. The Hague/Paris, 211-219.

Kotin, Michail L. (2003): Die werden-Perspektive und die werden-Periphrase im Deutschen. Historische Entwicklung und Funktionen in der Gegenwartssprache. Frankfurt (M.) (=Danziger Beiträge zur Germanistik 6).

LAZARD, GILBERT (2001): On the grammaticalization of evidentiality. In: Journal of Pragmatics 33:359-367.

Marschall, Matthias (1987): Paul wird in der Badewanne sitzen. Das Futur in der gesprochenen deutschen Sprache und ein Teilsystem der deutschen Verbformen. In: Deutsche Sprache 15:122-136.

Mortelmans, Tanja (2004): Grammatikalisierung und Subjektivierung: Traugott und Langacker revisited. In: Zeitschrift für Germanistische Linguistik 32:188-209.

NUYTS, JAN (2001): Epistemic Modality, Language and Conceptualization. Amsterdam. PALMER, Frank Robert (1986/2001): Mood and Modality. Cambridge.

RONCADOR, MANFRED VON (1988): Zwischen direkter und indirekter Rede: nichtwörtliche direkte Rede, erlebte Rede, logophorische Konstruktionen und Verwandtes. Tübingen (=Linguistische Arbeiten 192).

SMirnova, ElEna (2006): Die Entwicklung der Konstruktion würde + Infinitiv im Deutschen. Eine funktional-semantische Analyse unter besonderer Berücksichtigung sprachhistorischer Aspekte. Berlin/New York (=Studia Linguistica Germanica 82).

SocKA, ANNA (2006): würde mit Infinitiv - Grenzen der semantischen Kompositionalität. In: Cirko, LesŁAw / GRIMBERG, MARTIN (eds.): Phänomene im syntaktisch-semantischen Grenzbereich. Materialien der internationalen Linguistenkonferenz Karpacz 27.-29.09.2004. Dresden/Wrocław (=Beihefte zu Orbis Linguarum 47), 147-165.

- (2008): Die würde-Konstruktion - zwischen Indirektheit und Kontrafaktivität. In: Letnes, Ole / MAAGerø, Eva / VATER, Heinz (eds.): Modalität und Grammatikalisierung / Modality and Grammaticalization. Trier (=Fokus Linguistisch-Philologische Studien 34), 199-216.

SÜSKInd, PATRICK (1985): Das Parfum. Die Geschichte eines Mörders. Zürich.

Thieroff, Rolf (1992): Das finite Verb im Deutschen. Tempus - Modus - Distanz. Tübingen (=Studien zur deutschen Grammatik 40).

WEINRICH, HARALD (1993): Textgrammatik der deutschen Sprache. Mannheim.

WelKe, Klaus (2005): Tempus im Deutschen. Rekonstruktion eines semantischen Systems. Berlin/New York (=Linguistik - Impulse \& Tendenzen 13).

WIEMER, BJÖRN (2005): Conceptual affinities and diachronic relationships between epistemic, inferential and quotative functions (preliminary observations on lexical markers in Russian, Polish and Lithuanian). In: HANSEN, BJörn / KARLík, PETR (eds.): Modality in Slavonic languages. New perspectives. München (=Slavolinguistica 6), 107-131. 
Die Konstruktion würde+Infinitiv als evidentieller Ausdruck des Deutschen

- (2006): Particles, parentheticals, conjunctions and prepositions as evidentiality markers in contemporary Polish (a first exploratory study). In: Studies in Polish Linguistics 3:5-67.

WiERZBICKA, ANNA (2006): English: Meaning and Culture. Oxford.

WiLlett, ThOmas (1988): A crosslinguistic survey of the grammaticalization of evidentiality. In: Studies in Language 12:51-97.

Zifonun, Gisela et al. (eds.) (1997): Grammatik der deutschen Sprache. Berlin/New York. 